\title{
Inability of the Polyphasic Approach to Systematics To Determine the Relatedness of the Genera Xenorhabdus and Photorhabdus
}

\author{
F. A. RAINEY, ${ }^{1}$ R.-U. EHLERS, ${ }^{2}$ AND E. STACKEBRANDT ${ }^{1 *}$ \\ DSM-Deutsche Sammlung von Mikroorganismen und Zellkulturen GmbH, 38124 Braunschweig, ${ }^{1}$ \\ and Abteilung Biotechnologie und Biologischer Pflanzenschutz, Institut für Phytopathologie, \\ Christian-Albrechts-Universität, 24223 Klausdorf, ${ }^{2}$ Germany
}

\begin{abstract}
Comparative analysis of the genes coding for 16S rRNA of the type strains of Xenorhabdus and Photorhabdus species indicates the close phylogenetic relationship of these two genera. However, distance matrix analyses do not unambiguously separate the symbionts of entomopathogenic nematodes according to their assignment into different genera. When various 16S rRNA gene sequences from a selection of members of the gamma subclass of Proteobacteria and outgroup taxa were used, the intrageneric relationships of Xenorhabdus species and the positions of Photorhabdus luminescens and related species changed significantly.
\end{abstract}

The genus Photorhabdus has recently been described for a group of bioluminescent and facultatively anaerobic insectpathogenic strains that are symbionts of entomopathogenic nematodes belonging to the family Heterorhabditidae (3). The exclusion of Photorhabdus luminescens from the genus Xenorhabdus was based on low interspecies DNA reassociation values $(3,6,7,16)$ and the presence of distinguishing phenotypic $(2$, $3)$ and chemotaxonomic (16) properties. Thus, the genus $X e$ norhabdus was emended to contain only strains isolated from different entomopathogenic nematode species of the family Heterorhabditidae. None of the studies, however, were able to determine the intergeneric relatedness of these two taxa. Similarly, 16S rRNA cataloguing performed on two strains of $P$. (Xenorhabdus) luminescens and one $X$. nematophilus strain (5) was carried out not to determine the possible phylogenetic heterogeneity of the genus Xenorhabdus, but to investigate whether these organisms belong to the family Enterobacteriaceae. In this study, we determined the 16S rRNA gene (16S rDNA) sequences of all type strains of both genera, and we discuss the problem of unambiguous phylogenetic placement of organisms.

\section{MATERIALS AND METHODS}

Strains investigated. Xenorhabdus nematophilus DSM $3370^{\mathrm{T}}, X$. beddingii DSM $4764^{\mathrm{T}}, X$. poinarii DSM $4768^{\mathrm{T}}, X$. bovienii $4766^{\mathrm{T}}$, and Photorhabdus luminescens DSM $3368^{\mathrm{T}}$ and DSM 3369 are deposited in the DSM-German Collection of Microorganisms and Cell Cultures. P. luminescens $\mathbf{H S H} 2$ was isolated by R.-U. Ehlers from the nematode Heterorhabditis megidis HSH2. All strains were cultivated on medium 423 as indicated in the DSM catalogue of strains.

Analysis of 16S rDNA. Extraction of genomic DNA and amplification of the $16 S$ rDNA were carried out as described previously (11). PCR products were sequenced directly by using the Taq DyeDeoxy Terminator Cycle sequencing kit (Applied Biosystems) according to the manufacturer's protocol. The sequence reaction mixtures were electrophoresed by using the Applied Biosystems 373A DNA sequencer.

Phylogenetic analysis. 16S rDNA sequences were compared with the existing $16 \mathrm{~S}$ rDNA database of members of the family Enterobacteriaceae and other members of the gamma subclass of Proteobacteria (9). Dissimilarity values were transformed into phylogenetic distance values that compensate for multiple substitutions at any given site in the sequence (8). Phylogenetic trees were

\footnotetext{
* Corresponding author. Mailing address: DSM-Deutsche Sammlung von Mikroorganismen und Zellkulturen $\mathrm{GmbH}$, Mascheroder Weg 1b, 38124 Braunschweig, Germany. Phone: 495312616352. Fax: 5312616 418. Electronic mail address: stackebrandt@venus.gbfbraunschweig.d400.de.
}

reconstructed by applying additive treeing methods, such as neighbor joining (12) and the algorithm of De Soete (4), using the corrected dissimilarity values. One thousand bootstrap values for 920 polymorphic sites were calculated to test the stability of the neighbor-joining tree.

Nucleotide sequence accession numbers. The 16S rDNA sequences have been deposited at EMBL under accession no. X82248 to X82254.

\section{RESULTS}

The almost complete sequences of the seven strains of $X e$ norhabdus and Photorhabdus species were analyzed. Binary $16 \mathrm{~S}$ rDNA similarities for the Xenorhabdus strains ranged between 96.0 and $97.7 \%$, while the values for these organisms and $P$. luminescens ranged between 94.1 and $96.6 \%$. With 92.3 to $95.2 \% 16 \mathrm{~S}$ rDNA similarity, the membership of Xenorhabdus and Photorhabdus in the family Enterobacteriaceae is in accord with previous findings (5). Proteus vulgaris can be considered the nearest phylogenetic neighbor (between 93.5 and $95.1 \%$ similarity). Other members of this subclass, including Vibrio parahaemolyticus, selected as an out-group member of the gamma subclass of Proteobacteria, have significantly less similarity, mostly $<90 \%$ (data not shown).

Independently of the number of generic affiliation of outgroup reference organisms, two of the most widely used algorithms for generating phylogenetic trees from dissimilarity values, i.e., the distance matrix methods of Saitou and Nei (12) and De Soete (4), consistently placed strains of Xenorhabdus and Photorhabdus on a single line of descent, separate from other members of the family Enterobacteriaceae. The clustering is supported by $100 \%$ significance as determined by bootstrap analysis. The number of signature nucleotides in the $16 \mathrm{~S}$ rDNAs of Photorhabdus and Xenorhabdus strains is small, i.e., at positions 19 to 916 (C-U versus A-U, found in other enterobacteria) and positions 154 to 167 (C-G versus U-A, found in most other enterobacteria). A few signature nucleotides are shared with Proteus vulgaris, such as those at positions 320 to 333 (G-C versus A-U), 576 to 647 (A-U versus U-A), 577 to 646 (A-U versus G-C), and 682 to 707 (Y-G versus G-C). The $\mathrm{G}+\mathrm{C}$ contents of $16 \mathrm{~S}$ rDNAs of both genera of the symbionts of entomopathogenic nematodes are about 2 to $3 \%$ higher (55.1 to $55.5 \%)$ than those of other members of the Enterobacteriaceae (52.8 to $53.4 \%)$.

With a selection of nine species of the family Enterobacteriaceae and $V$. parahaemolyticus used as reference organisms, a neighbor-joining tree was generated (Fig. 1). In contrast to the 


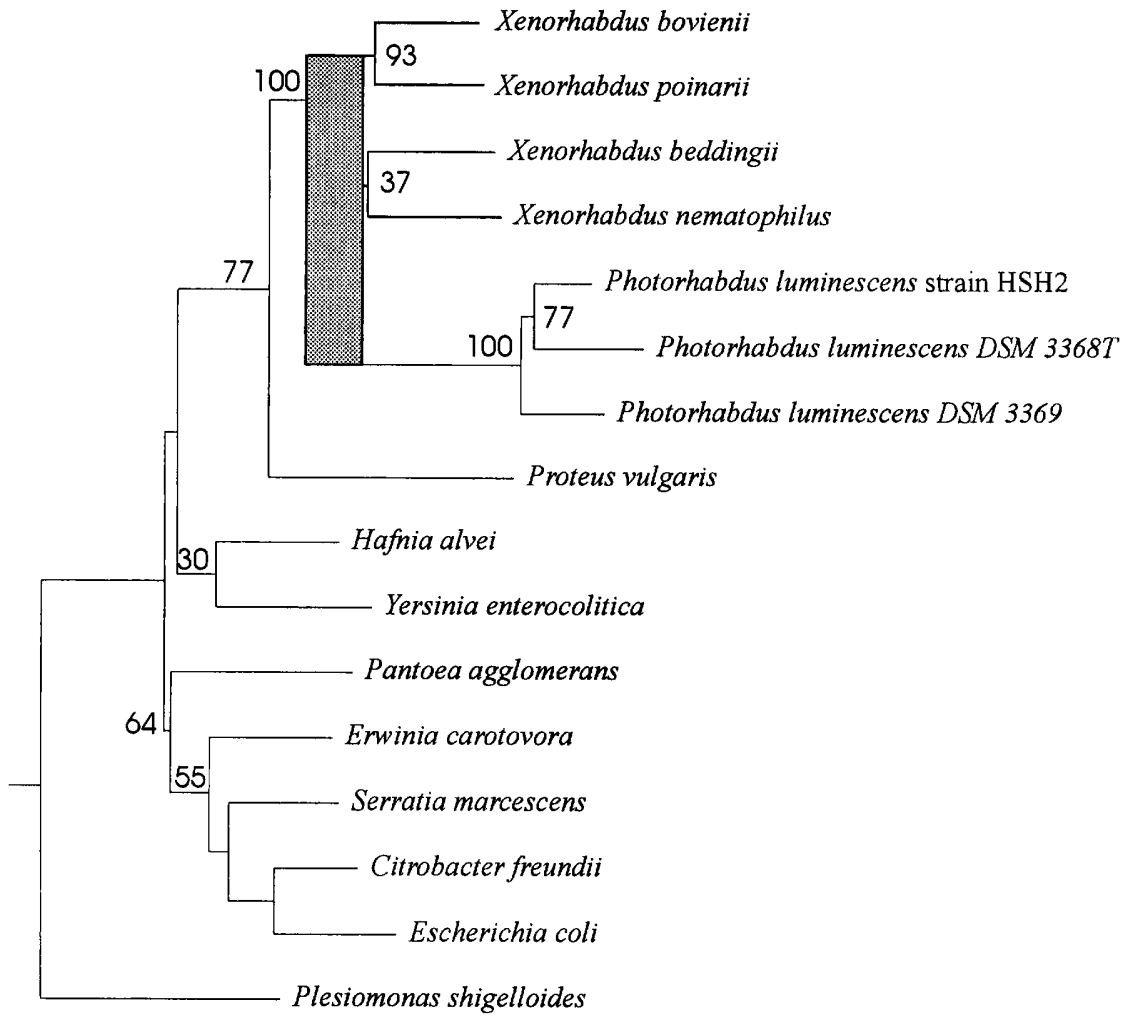

0.05

FIG. 1. Neighbor-joining tree showing the phylogenetic relationships among members of the genera Xenorhabdus and Photorhabdus and members of the family Enterobacteriaceae. V. parahaemolyticus served as a root. The region in which the order of branching points leading to species of Xenorhabdus and Photorhabdus cannot be determined with confidence is indicated (shaded box). The bar represents five nucleotide substitutions per 100 nucleotides.

distinctly lower $16 \mathrm{~S}$ rDNA similarity of about $2 \%$, the three strains of $P$. luminescens do not cluster apart from Xenorhabdus strains but, rather, branch off from within the radiation of strains of the latter genus. While $X$. nematophilus and $X$. beddingii form a pair and $X$. bovienii and $X$. poinarii form a pair of distantly related species, supported by bootstrap values of 37 and $93 \%$, respectively, $X$. poinarii and $X$. bovienii branch more deeply than the three $P$. luminescens strains. This branching pattern is not in accord with the bootstrap analysis, which indicated that, to the exclusion of $P$. luminescens, the type strains of Xenorhabdus clustered together in $65 \%$ of simulated trees. The same topology was obtained when the tree was generated by using the algorithm of De Soete (4). To investigate whether the number and origin of homologous sequences from various taxa of the gamma subclass influence the branching point of the Xenorhabdus and Photorhabdus species, 15 different sequence sets were analyzed by the two distance matrix algorithms. The number of sequences ranged from 8 to 40 , and the selection of sequences included those of the symbiotic bacteria of the genus Buchnera (10) as well as those of the most deeply branching taxa of this subclass, i.e., sequences of species of Nitrosomonas, Coxiella, Xanthomonas, Chromatium, and Ectothiorhodospira. With two exceptions, in which the two genera investigated form phylogenetic sister groups, $P$. luminescens grouped within the radiation of Xenorhabdus species. This was independent of whether only the type strain of $P$. luminescens or all three strains of this species were included in the analysis. In most of these cases, $P$. luminescens clustered with the pair $X$.
nematophilus-X. beddingii, and $X$. bovienii clustered most deeply. This situation is similar to the one depicted in Fig. 1. The exceptions are those cases in which, in addition to the sequences of Xenorhabdus and Photorhabdus strains, randomly selected sequences of either only Escherichia coli or of all enterobacteria, three sequences of Buchnera strains, and one representative each of the genera Vibrio and authentic Pseudomonas were used (not shown). In order to demonstrate the inability of the algorithms and reference sequences used to reliably depict the relatedness between members of the genera Xenorhabdus and Photorhabdus, the region of uncertain branching points is indicated in Fig. 1.

\section{DISCUSSION}

Justification for the exclusion of $X$. luminescens from the genus Xenorhabdus and the description of Photorhabdus to accommodate this species was based on results of DNA-DNA pairing experiments, as well as chemotaxonomic and phenotypic characteristics. The intrageneric relatedness of Xenorhabdus species has been determined previously by different DNA-DNA reassociation methods $(3,6,7,16)$. In all of these studies, DNA relatedness determined for the type strains of $X$. nematophilus and $X$. luminescens was $<20 \%$, while the similarity between the type strains of the other Xenorhabdus species ranged between 20 and $40 \%$. As DNA reassociation values for use in genus delineation are lacking, and low similarity values alone do not justify the dissection of a genus, further evidence 
for the exclusion of $X$. luminescens was needed. While the ubiquinone composition and the DNA G $+\mathrm{C}$ content were not able to discriminate between these two taxa, differences in the major cellular fatty acids had been reported (16). However, the strains of $X$. luminescens differed from the other members of the genus only in having a slightly higher content of i-15 branched fatty acids. Results of a numerical taxonomic study showed that the symbionts of Heterorhabditis spp., $X$. luminescens strains, form a discrete cluster regardless of the datum set and the clustering strategy used. Thus, the phenetic data are in accordance with the DNA pairing study, the fatty acid analysis, and the $16 \mathrm{~S}$ rDNA analysis. Strains of $X$. luminescens were linked most closely to the Xenorhabdus strains isolated from Steinernema organisms of Australian origin (group V as defined by Akhurst and Boemare [1]) which were described as $X$. beddingii. In three of four numerical analyses $X$. luminescens and $X$. beddingii formed a sister taxon to strains of $X$. bovienii, and in only one analysis strains of $X$. luminescens branched earlier than strains of the other species of Xenorhabdus. It thus appears that the exclusion of $X$. luminescens from the genus $X e n o r h a b d u s$ and the description of $P$. luminescens were done mainly on the basis of some phenotypic data in which strains of this species differed exclusively from strains of Xenorhabdus, such as presence of bioluminescence, positive catalase reaction, assimilation of arbutin, lack of DL-lactate production, and the host nematode. However, not all strains of $P$. luminescens are luminescent (1), and some strains closely related to $X$. bovienii are also catalase positive.

We do not want to question the validity of the transfer of $X$. luminescens to a new genus. The 16S rDNA analysis reflects the situation of the numerical phenetic analysis closely in that the relationship of $P$. luminescens to the species of Xenorhab$d u s$ depends heavily on the database used. Thus, if one agrees to this taxonomic conclusion, the two genera can nevertheless be considered very closely related, to the extent that the degree of genomic differences observed between the type species of the two genera is not significantly higher than that found between the species of Xenorhabdus.

The finding that the selection of reference organisms influences tree topologies quite significantly has been observed previously $(14,17)$. In general, every new sequence which is different from those in the existing datum set provides additional data on the information content of individual sequence positions as well as on the characteristics defining phylogenetic groups. The inclusion of this additional information may change the tree locally or even globally. This effect is recognized by those who construct phylogenetic trees from sequence data. Of the most obvious parameters, we can exclude a bias introduced by differences in the base composition of rDNA, as all Xenorhabdus and Photorhabdus species have very similar $\mathrm{G}+\mathrm{C}$ values. Bias introduced by partial sequences (13) can be excluded, as almost complete sequences have been compared. However, we have noted that Photorhabdus species may be subjected to a slightly higher evolutionary rate ("fast clock" or tachytelic behavior). Support for this hypothesis originates from the finding that the level of similarity between these strains and the reference organism is about $2 \%$ lower than that found for Xenorhabdus species and the reference organisms (which should be very similar in comparison with out-group sequences) (15). At the nucleotide level, this hypothesis is supported by the lack of several absolutely unique positions in the sequences of $P$. luminescens which are conserved for members of the gamma subclass of Proteobacteria (nucleotides 1001 to 1002 pairing with nucleotides 1038 to 1039 and nucleotides 1006 to 1009 pairing with nucleotides 100 to 1023 ; bp 1254 to 1283). Furthermore, strains of $P$. luminescens show greater variation in the nucleotide composition of hypervariable regions than is found among the type strains of the Xenorhabdus species.

The strategy that should be considered next is to increase the stability of the tree by adding more sequences of members of the two species, especially more strains of $P$. luminescens. If, as expected, $P$. luminescens strains are slightly more rapidly evolving, then this should be reflected and detectable in a greater variation of those nucleotide positions considered to be conserved in neighboring taxa.

\section{REFERENCES}

1. Akhurst, R. J., and N. E. Boemare. 1988. A numerical taxonomic study of the genus Xenorhabdus (Enterobacteriaceae) and proposed elevation of the subspecies of $X$. nematophilus to species. J. Gen. Microbiol. 134:1835-1845.

2. Boemare, N. E., and R. J. Akhurst. 1988. Biochemical and physiological characterization of colony form variants in Xenorhabdus spp. (Enterobacteriaceae). J. Gen. Microbiol. 134:751-761.

3. Boemare, N. E., R. J. Akhurst, and R. G. Mourant. 1993. DNA relatedness between Xenorhabdus spp. (Enterobacteriaceae), symbiotic bacteria of entomopathogenic nematodes, and a proposal to transfer $X$. luminescens to a new genus, Photorhabdus gen. nov. Int. J. Syst. Bacteriol. 43:249-255.

4. De Soete, G. 1983. A least squares algorithm for fitting additive trees to proximity data. Psychometrika 48:621-626.

5. Ehlers, U.-R., U. Wyss, and E. Stackebrandt. 1988. 16S rRNA cataloging and the phylogenetic position of Xenorhabdus. Syst. Appl. Microbiol. 10:121-125.

6. Farmer, J. J., J. H. Jorgensen, P. A. Grimont, R. J. Akhurst, G. O. Poinar, E. Ageron, G. V. Pierce, J. A. Smith, G. P. Carter, K. L. Wilson, and F. W. Hickman-Brenner. 1989. Xenorhabdus luminescens (DNA hybridization group) from human clinical specimens. J. Clin. Microbiol. 27:1594-1602.

7. Grimont, P. A., A. G. Steigerwalt, N. E. Boemare, F. W. Hickman-Brenner, C. Deval, F. Grimont, and D. Brenner. 1984. Deoxyribonucleic acid relatedness and phenotypic study of the genus Xenorhabdus. Int. J. Syst. Bacteriol. 34:378-388.

8. Jukes, T. H., and C. R. Cantor. 1969. Evolution of protein molecules, p. 21-132. In H. N. Munro (ed.), Mammalian protein metabolism. Academic Press, New York.

9. Larsen, N., G. J. Olsen, B. L. Maidak, M. McCaughey, J. R. Overbeek, T. J. Macke, T. L. Marsh, and C. R. Woese. 1993. The ribosomal database project. Nucleic Acids Res. 21:3021-3023.

10. Munson, M. A., P. Baumann, and M. G. Kinsey. 1991. Buchnera gen. nov. and Buchnera aphidicola sp. nov., a taxon consisting of the mycetocyteassociated, primary endosymbionts of aphids. Int. J. Syst. Bacteriol. 41:566568.

11. Rainey, F. A., M. Dorsch, H. W. Morgan, and E. Stackebrandt. 1992. 16S rDNA analysis of Spirochaeta thermophila: its phylogenetic position and implications for the systematics of the order Spirochaetales. Syst. Appl. Microbiol. 15:197-202.

12. Saitou, N., and M. Nei. 1987. The neighbor-joining method: a new method for reconstructing phylogenetic trees. Mol. Biol. Evol. 4:406-425.

13. Stackebrandt, E., W. Liesack, and D. Witt. 1992. Ribosomal RNA and ribosomal DNA sequence analyses. Gene 115:255-260.

14. Stackebrandt, E., and W. Ludwig. 1994. The importance of using outgroup reference organisms in phylogenetic studies: the Atopobium case. Syst. Appl. Microbiol. 17:39-43.

15. Stackebrandt, E., and C. R. Woese. 1981. The evolution of prokaryotes, p. 1-31. In M. J. Carlile, J. F. Collins, and B. E. B. Moseley (ed.), Molecular and cellular aspects of microbial evolution. Cambridge University Press, Cambridge.

16. Suzuki, T., S. Yamanaka, and Y. Nishimura. 1990. Chemotaxonomic study of Xenorhabdus species-cellular fatty acids, ubiquinone and DNA-DNA hybridization. J. Gen. Appl. Microbiol. 36:393-401.

17. Woese, C. R., L. Achenbach, P. Rouviere, and L. Mandelco. 1991. Archaeal phylogeny: reexamination of the phylogenetic position of Archaeoglobus fulgidus in the light of certain composition-induced artifacts. Syst. Appl. Microbiol. 14:364-371. 